\title{
Identify the critical regions and switching/failure mechanisms in non-filamentary RRAM (a-VMCO) by RTN and CVS techniques for memory window improvement
}

\author{
J. Ma ${ }^{(1)}$, Z. Chai ${ }^{(1)}$, W. Zhang ${ }^{(1)}$, B. Govoreanu ${ }^{(2)}$, J. F. Zhang ${ }^{(1)}$, Z. Ji ${ }^{(1)}$, B. Benbakhti ${ }^{(1)}$, G. Groeseneken ${ }^{(2)}$ and M. Jurczak ${ }^{(2)}$ \\ ${ }^{(1)}$ Department of EEE, Liverpool John Moores University, Liverpool L3 3AF, UK ${ }^{(2)}$ IMEC, Leuven B3001, Belgium
}

\begin{abstract}
Non-filamentary RRAM is a promising technology that features self-rectifying, forming/compliance-free, tight resistance distributions at both high and low resistance states (HRS/LRS). Direct experimental evidence for its physical switching \& failure mechanisms, however, is still missing, due to the lack of suitable characterization techniques. In this work, a novel method combining the random-telegraph-noise (RTN), constant-voltagestress (CVS) and time-to-failure Weibull plot is developed to investigate these mechanisms in the non-filamentary RRAM cell based on amorphous-Si/ $/ \mathrm{TiO}_{2}$. For the first time, the following key advances have been achieved: i) Switching mechanism by defect profile modulation in a critical interfacial region has been identified from defect locations extracted by RTN; ii) Defect profile in this region plays a critical role in device failure, leading to different Weibull distributions during negative (LRS) and positive (HRS) CVS; iii) Progressive formation of a conductive percolation path during electrical stress is directly observed due to defect generation in addition to pre-existing defect movement; iv) Optimizing the critical interfacial region significantly improves memory window and failure margin. This provides a useful tool for advancing the non-filamentary RRAM technology.
\end{abstract}

\section{Introduction}

Resistive switching memories (RRAM) attracted extensive attentions as an emerging memory technology. There are two types of oxygen ion/vacancy based RRAMs: (1) filamentary RRAM: Switching is through the modification of a conductive filament, which leads to long HRS/LRS retention tails (e.g. $\mathrm{HfO}_{2}$, [1-2]) (Fig.1a\&c); (2) Non-filamentary RRAM: It features selfrectifying, forming/compliance-free, low $\mu \mathrm{A}$ operation, tighter HRS/LRS distributions with little tail-bits shifting issues (e.g. $\mathrm{TiO}_{2} / \mathrm{TaO}_{\mathrm{x}}$ [3], a-VMCO [4,5]). It has been proposed that its switching is through the uniform defect profile modulation (Fig.1b\&d) [4], however, direct experimental evidence is still missing and its failure mechanism is not known. In this work, a novel method has been developed combining RTN, CVS and Weibull plot, which, for the first time, enables the identification of its switching and failure mechanisms at defect level by directly observing the profile modulation of pre-existing defects and the progressive formation of a conductive percolation path by the addition of generated defects. The unique failure characteristics of a-VMCO can be explained by a proposed model. Identifying the key roles of the critical interfacial region in switching/failure provides guidance for further improving non-filamentary RRAM.

\section{Device and operation}

A TEM image of the device structure and the DC I-V curves are shown in Fig.1e\&f, respectively [4]. Device details are given in Table 1. The a-VMCO cell features analog switching behavior. It RESETs at a positive top electrode (TE) voltage and SETs at a negative bias. Increasing Vreset is desirable for enhancing the memory window, but this will soon cause breakdown in devices with unoptimized process (W1). By improving the critical regions responsible for switching/failure through process optimization (W2\&W3), we will demonstrate that the memory window and failure margin can be significantly improved (Fig.1g).

\section{RTN \& CVS Characterization}

\section{A. RTN and defect profile modulation by switching}

In our earlier work [2], RTN in $\mathrm{HfO}_{2}$ RRAM has been used to extract the profile of defect's spatial and energy locations, $\mathrm{X}_{\mathrm{T}}$ \& $\mathrm{E}_{\mathrm{T}}$, from its mean capture/emission time constant dependence on the applied bias [6-8]. By adapting this method to a-VMCO, RTN can be clearly observed and defects are detected in both $\mathrm{TiO}_{2}$ and a-Si layers (Fig.2). Defects profiles in the vertical direction extracted after switching to LRS (o) and HRS (o) are compared in Fig.3a\&b. At HRS, there is a defect-'less' region at each side of the a-Si/ $/ \mathrm{TiO}_{2}$ interface (IL). At LRS, however, it is not observed at the $\mathrm{TiO}_{2}$ side; hence this is where the defect profile modulation occurs predominantly. The total width of defect-'less' regions is closely correlated with resistance, as it becomes wider at HRS and narrower at LRS, providing, for the first time, direct experimental evidence for the proposed switching mechanism (Fig.1b) [4-5]. Note that this defect profile modulation is caused by the movement of pre-existing defects, which have uniform spatial distribution in the lateral direction (Fig.1b), because of its forming-free and area-dependent non-filamentary switching characteristics as shown in refs. $[4,5]$.

\section{B. Time-to-failure during CVS and its Weibull plot}

In order to characterize the device failure, CVS is applied on TE and time-to-breakdown, $\mathrm{t}_{\mathrm{BD}}$, is examined with Weibull plot. It exhibits a single slope for positive $\mathrm{CVS}$ at $\mathrm{V}_{\mathrm{BD}}=+6.6 \mathrm{~V}$ (Fig. 4a), but a bimodal behavior with two slopes for negative CVS at a much smaller $V_{\mathrm{BD}}=-3.5 \mathrm{~V}$ (Fig.4b). This significant polaritydependence of $\mathrm{V}_{\mathrm{BD}}$ and Weibull slopes have not been observed for high-k dielectric stacks used in MIM capacitor or MOSFETs (e.g. Fig.5) [9-13]. The cell current is also monitored against stress time during CVS, showing two stages before reaching breakdown: in $1^{\text {st }}$ stage, current reduces at positive CVS (Fig.6a), but is stable at negative CVS (Fig.6b). Large fluctuations are observed in $2^{\text {nd }}$ stage in both cases. In order to explain these differences, defect profiles during CVS will be obtained next by combining RTN and CVS measurements.

\section{Combination of RTN and CVS}

RTN measurements are inserted at certain intervals during CVS (Fig.7) to monitor the gradual changes in defect profiles at different CVS stages. RTNs with only small amplitudes $(<5 \%)$ are observed in $1^{\text {st }} \mathrm{CVS}$ stage (also in fresh devices after switching to either HRS or LRS, not shown), whilst both small and large RTNs $(>10 \%)$ are observed in $2^{\text {nd }}$ CVS stage (Fig.8a-d). This suggests that in addition to the profile modulation of pre-existing defects during set/reset and in $1^{\text {st }}$ stage, additional defects are generated by stress in $2^{\text {nd }}$ stage, leading to the large amplitude of RTN and 
current fluctuations, and breakdown. The same defects can be detected by immediately repeated RTN measurements in both $1^{\text {st }}$ and $2^{\text {nd }}$ stages, confirming the effectiveness of the measurement (Fig.9). In the following, defect profiles during $1^{\text {st }}$ and $2^{\text {nd }}$ stages of both CVS polarities will be examined, and a physical model for the failure mechanism will be proposed.

\section{Failure mechanisms}

\section{A. Defect profile during positive CVS}

The positive CVS bias resets a-VMCO to HRS. As shown in Fig.1b\&3b, switching to HRS is associated with two pre-existing defect-'less' regions, one at a-Si side and the other at $\mathrm{TiO}_{2}$ side of IL. Profile of pre-existing defects extracted during the $1^{\text {st }}$ stage of positive CVS (Fig.3b) is shown as the orange-color background in Fig.10a-c. The current reduction in $1^{\text {st }}$ stage (Fig.6a) is caused by deeper reset under the positive CVS. Defects extracted from the large RTN signals ( $\bullet$ ) in $2^{\text {nd }}$ CVS stage are observed in the pre-existing defect-'less' (white) region in $\mathrm{TiO}_{2}$ at early $2^{\text {nd }}$ stage, gradually reaching the $\mathrm{TiO}_{2} / \mathrm{IL}$ interface (Fig.10a-c), confirming that they are caused by defects generation as they are absent in this region during normal switching. Defects generation is also observed in a-Si approaching its pre-existing defect-'less' region. This result suggests that the defect-'less' interfacial region plays a key role as the 'last-to-break' region. The wider pre-existing defect-'less' regions at HRS (Fig.1b \& 10d) result in a higher positive $\mathrm{V}_{\mathrm{BD}}$ with the potential drop across the $\mathrm{TiO}_{2} / \mathrm{a}-\mathrm{Si}$ stack $\mathrm{V}_{\text {stack }}=\mathrm{V}_{\mathrm{CVS}}-\mathrm{V}_{\mathrm{FB}}=5.6 \mathrm{~V}$ (Fig. 2).

\section{B. Defect profile during negative CVS}

The negative CVS bias sets a-VMCO to LRS, and the $\mathrm{TiO}_{2}$ side of IL is occupied by the pre-existing defects (Fig.1b\&3). Profile of the pre-existing defects extracted during the $1^{\text {st }}$ stage of negative CVS (Fig.3b) is shown as the light-blue background in Fig.11a-c. Defects causing large RTNs in $2^{\text {nd }}$ stage evolve in a way similar to that under positive CVS: they are observed in $\mathrm{TiO}_{2}$ gradually approaching the $\mathrm{TiO}_{2} / \mathrm{IL}$ interface, and also in a-Si approaching the pre-existing defect-'less' region. This eventually leads to the formation of a percolation path across the $\mathrm{TiO}_{2} / \mathrm{a}-\mathrm{Si}$ stack, as can be seen clearly in the case after device soft BD (Fig.12). The narrower defect-'less' IL/a-Si region leads to a smaller negative $\mathrm{V}_{\mathrm{BD}}$ with the potential drop across the $\mathrm{TiO}_{2} / \mathrm{a}-\mathrm{Si}$ stack $V_{\text {stack }}=V_{C V S}-V_{F B}=-4.5 V$. Moreover, these devices are in the region 1 of its bimodal Weibull plot (Fig.4b).

Devices in region 2 of the bimodal Weibull plot for -CVS shows a stepper distribution at short $t_{\mathrm{BD}}$ (Fig.4b). Defect profiles in these devices are also extracted (Fig.13), and a percolation path is formed after short stress times, leading to their early failure. It should be emphasized that there is no device breakdown observed when stressed under $+\mathrm{CVS}$ at $\mathrm{V}_{\text {stack }}=4.5 \mathrm{~V}$ for $10 \mathrm{ks}$, suggesting the interfacial region becomes significantly more robust at HRS.

\section{Physical model for the failure mechanism}

Based on the above results and analysis, we propose a device failure model as illustrated in Fig.14a\&b. The failure of a-VMCO cell is a combined result of three factors: (1) the profile of pre-existing defects that can be modulated in the RRAM dielectric stack between HRS and LRS, unlike that in the classical gate dielectric of MOSFETs; (2) the conductive percolation path formed by defect generation during stress, which is affected by the pre-existing defects modulation; (3) quality of the $\mathrm{TiO}_{2} / \mathrm{a}-\mathrm{Si}$ interfacial region.

The two-slope bimodal distribution was also reported for some small-area high-k/SiON stacks in MOSFETs at both bias polarities and has been explained by the substantially higher defect generation rates (DGR) in either the high-k layer [9] or its grain boundary (GD) $[11,13]$. DGR can also explain the bimodal distribution in Fig.4b: the $\mathrm{TiO}_{2}$ side of IL is occupied by pre-existing defects under -CVS at LRS (Fig.3b), and has much higher DGR than the defect-'less' IL/a-Si region. The polarity dependence of the pre-existing defect distribution in Fig.14a\&b, however, is unique to the $\mathrm{TiO}_{2}$ layer in a-VMCO. The removal of pre-existing defects in the $\mathrm{TiO}_{2} / \mathrm{IL}$ region under + CVS at HRS reduces its DGR and the smaller difference in DGR for the two layers leads to the single slope in Fig.4a. To support this explanation, the Weibull plot of the Metal/a-Si/Metal devices [14] shows that the single a-Si layer has a similar single slope when the $\mathrm{TiO}_{2}$ layer was absent (Fig.15).

\section{Memory window improved by critical IL regions}

According to the above failure model, improving IL quality should reduce the DGR in the critical IL region, leading to better device performance. Indeed, an improved overall processing condition for both $\mathrm{TiO}_{2}$ and a-Si in W2/P2 lead to larger memory windows and failure margins as shown in Fig.1g\&16. This is consistent with $t_{\mathrm{BD}}$ improvement of W2 under both \pm CVS (Fig. 17a\&b). Interestingly, further improvement of $\mathrm{TiO}_{2} / \mathrm{IL}$ by a specific treatment prior to $\mathrm{TiO}_{2}$ deposition in W3 leads to a further improvement only in region 2 under -CVS (Fig.17b), supporting that the higher DGR in the $\mathrm{TiO}_{2}$ side of IL $[9,11]$ under - CVS at LRS is responsible for region 2. This is further supported in Fig.18. W1\&2\&3 have similar current fluctuation generation and failure path formation process, but at much longer stress times in $\mathrm{W} 2$ and $\mathrm{W} 3$ due to the lower $\mathrm{DGR}$ in $\mathrm{TiO}_{2} / \mathrm{IL}$ region with better quality, suggesting that exploring new IL material/process may facilitate even further improvement.

\section{Conclusions}

A novel method combining RTN, CVS and Weibull plot has been developed for non-filamentary RRAM device. For the first time, its defect profiles have been extracted by RTN, which are clearly correlated to resistance switching and to different time-tofailure Weibull distributions. Identification of defect generation during CVS enables the observation of progressive formation of the conductive percolation path leading to breakdown. This work provides insights for further improving the performance of nonfilamentary RRAM through identifying the roles of specific critical IL regions, assisting in advancing the RRAM technology.

Acknowledgement: Supported by EPSRC of UK (Grant no.: EP/ M006727/1) and fruitful discussions with colleagues at imec.

\section{References}

[1] B. Govoreanu, et al, IEDM, 2011. [2] Z. Chai, et al, VLSI Symp. Tech., 2016. [3] I. T. Wang. et al, IEDM, 2014. [4] B. Govoreanu, et al, VLSI Symp. Tech., 2015. [5] B. Govoreanu, et al, VLSI Symp. Tech., 2016. [6] M. J. Kirton, et al, Adv.s in Physics, vol. 38, no. 4, 1989. [7] C. M. Chang, et al, IEDM, 2008. [8] J. Chen, et al, VLSI Symp. Tech., 2014. [9] T. Nigam, et al, IRPS, 2009. [10] F. Mondon, Microele. Reliab., 2003. [11] N. Raghavan, et al, EDL, vol.32, no.1, 2011. [12] E. Wu, et al, IEDM, 2013. [13] K. S.Yew, et al EDL, vol.33, no.2, 2012. [14] L. Zhang, et al, IEDM, 2014. 


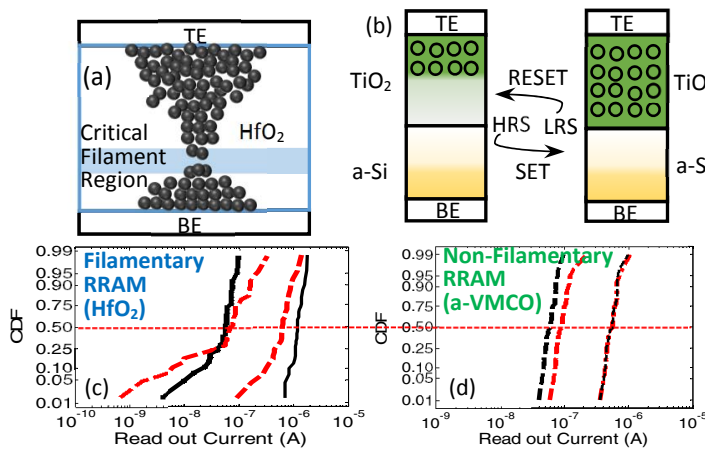

Fig.1 RRAM: (a) filamentary $\left(\mathrm{HfO}_{2}\right)$; (b) nonfilamentary (a-VMCO); (c) \& (d) Read current distributions before (black) and after (red) the retention test for (a) \& (b), respectively [4].

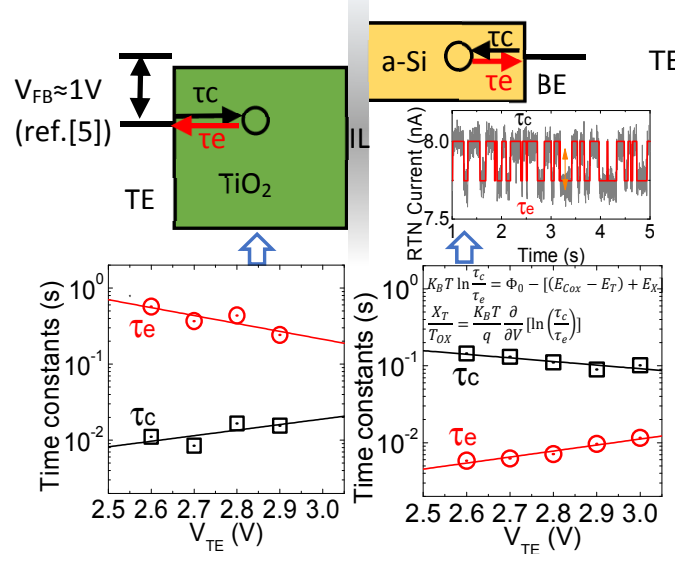

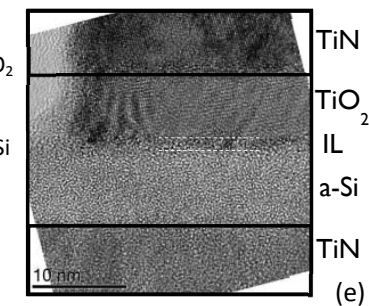

Fig.1 (e) TEM of a-VMCO RRAM, a $\sim 1-\mathrm{nm} \mathrm{SiO}_{\mathrm{x}}$ interfacial layer (IL) is formed between amorphous $\mathrm{Si}$ and anatase-phase $\mathrm{TiO}_{2}$ by the end of process $[4,5]$.
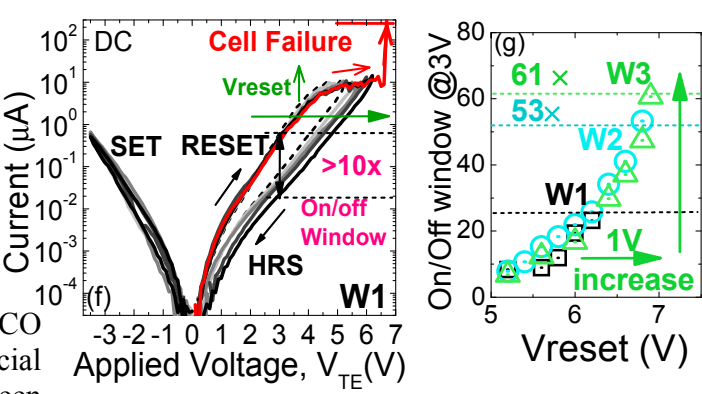

Fig.1 (f) DC I-V curves of a-VMCO cell (W1) at different Vreset. (g) Improved IL quality in W2 and $\mathrm{W} 3$ can enhance the memory window (Iread@3V) and failure margin significantly.
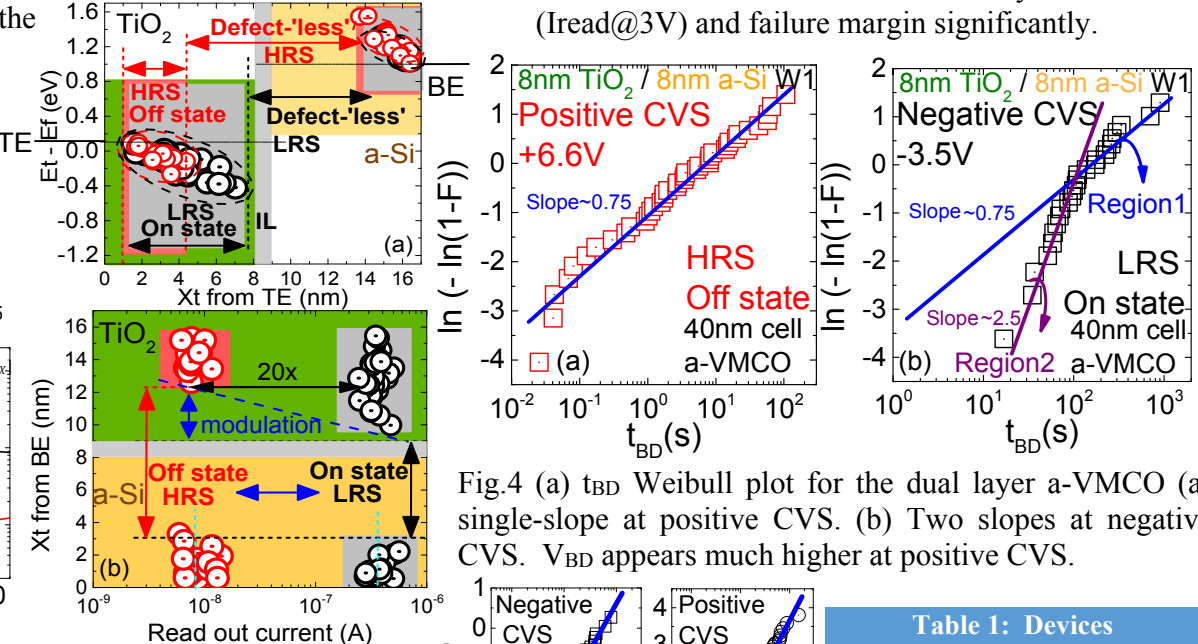

Fig.4 (a) tBD Weibull plot for the dual layer a-VMCO (a) single-slope at positive CVS. (b) Two slopes at negative CVS. VBD appears much higher at positive CVS.

Fig.2 Defect location is extracted from Fig.3 Extracted defect profiles at LRS/HRS $\overparen{\Psi}_{-1}$ the dependence of RTN's mean time (a) vs. distance from TE, and (b) vs. Iread. constants on bias $V_{T E}[2,6-8]$. Defects Note that this defect profile does not provide can be found in both $\mathrm{TiO}_{2}$ and a-Si.

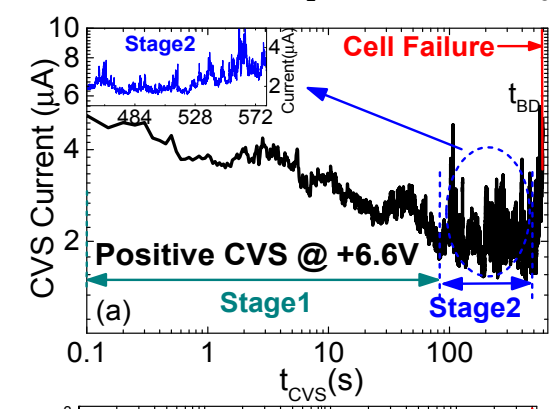
mation on the actual defect density [2] Defect profile modulation predominantly occurs in a critical region in $\mathrm{TiO}_{2}$ near IL.

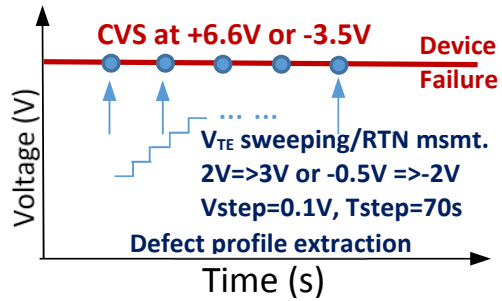

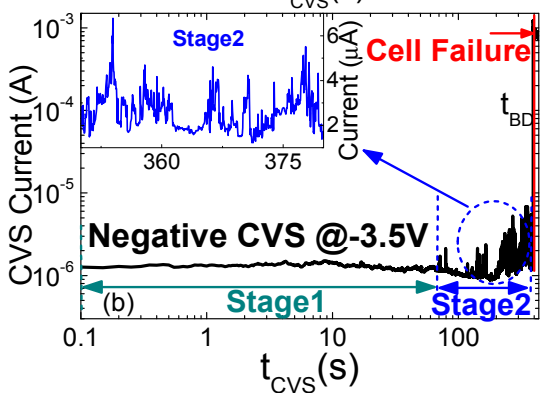

Fig.6 I-t plots during (a) positive CVS after reset at $+6.6 \mathrm{~V}$ and (b) negative CVS after set at $-3.5 \mathrm{~V}$. Stage 1 and Stage 2 are observed in both cases before device failure. CVS current reduction during $1^{\text {st }}$ stage in (a) is due to deeper reset under positive CVS. It is not observed in (b) due to the faster SET speed in a-VMCO [4].
Fig7. CVS and RTN measurement procedure for defect profile extraction. $\mathrm{V}_{\mathrm{TE}}$ during the RTN sweeping has the same polarity as CVS stress.
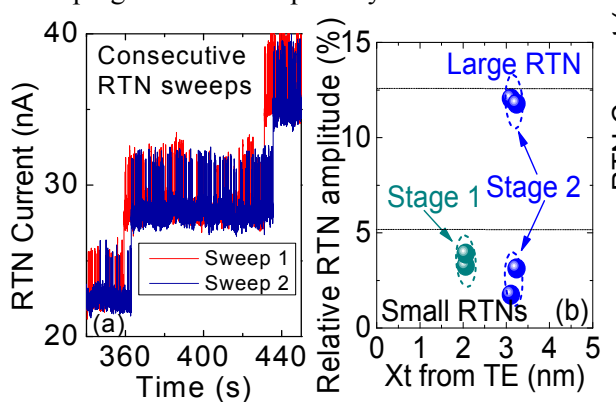

Fig.9 Same defects are measured by consecutive RTN sweeps as shown by (a) similar RTN signals, and (b) same defect locations and RTN amplitude in both Stage 1 and Stage 2.
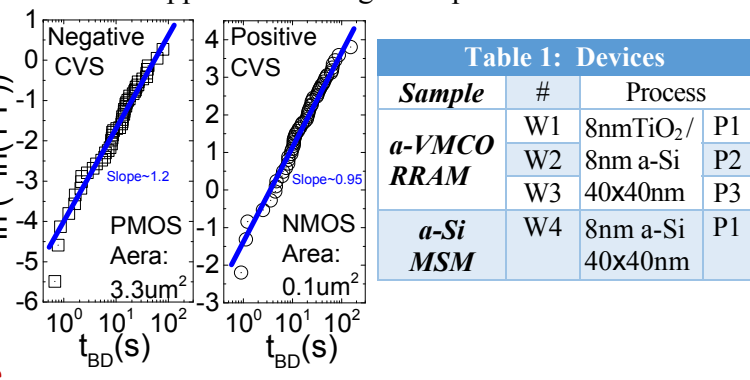

Fig5. $t_{\mathrm{BD}}$ Weibull plot for $\mathrm{SiO}_{2} / \mathrm{HfO}_{2}$ in $\mathrm{CMOS}$ device [9], similar distribution is observed at negative \& positive CVS.

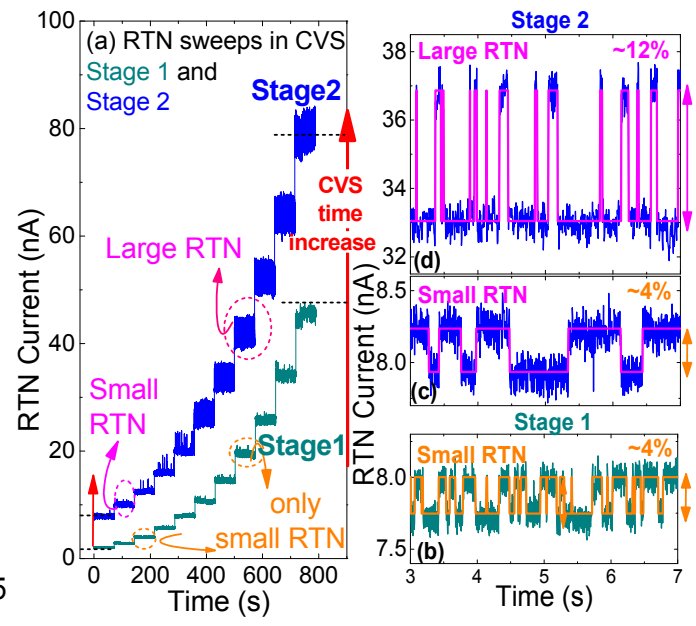

Fig.8 (a) I-V sweeps during RTN measurements in CVS Stage 1 \& Stage 2 as shown in Fig.6. (b) Only small RTNs in Stage 1. Both (c) small and (d) large RTNs in Stage 2. Large RTNs are caused by (or related to) defects generation in the percolation path. 
Case 1: Positive CVS @+6.6V, HRS, Off State

Positive CVS time increase
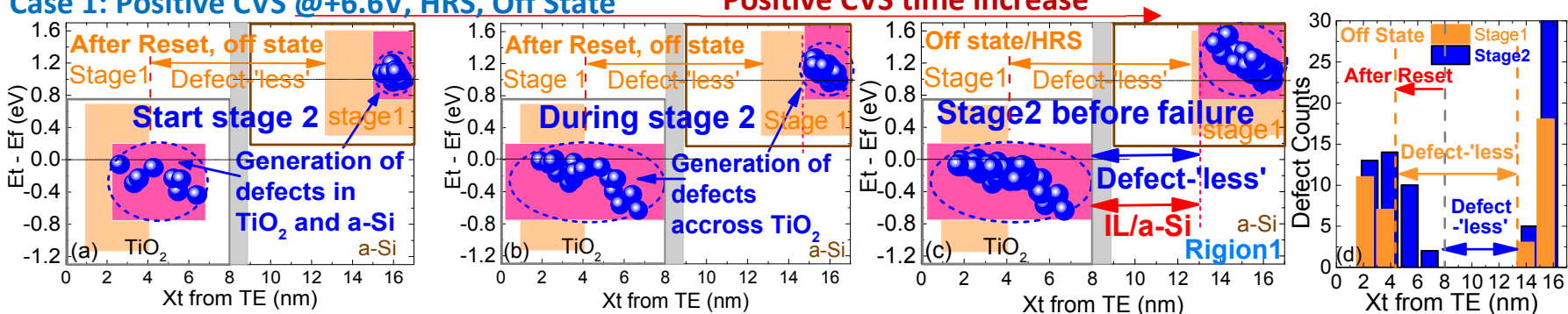

Fig10 Progressive percolation path formation by defects generation at the (a) start, (b) middle, (c) end of $2^{\text {nd }}$ stage during positive CVS, in addition to pre-existing defects observed during Stage 1. (d) The narrower defect-'less' region in $2^{\text {nd }}$ stage is the most robust region observed before BD.

Case 2: Negative CVS @-3.5V, LRS, On State

Negative CVS time increase
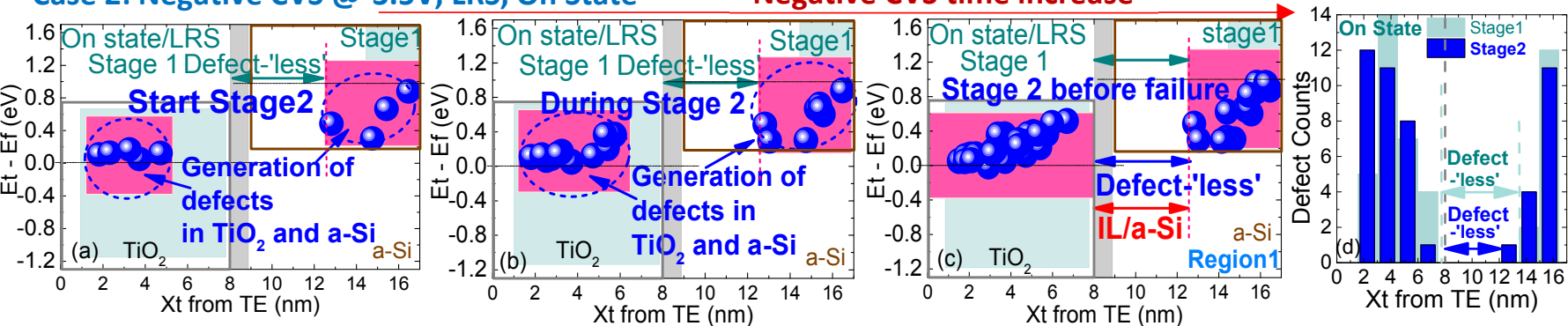

Fig11 (a-c) Progressive percolation path formation by defects generation during $2^{\text {nd }}$ stage of negative CVS, in addition to pre-existing defects observed during Stage 1. (d) Defects generation in the most robust defect-'less' IL/a-Si region can be observed after the soft BD as shown in Fig.12.

Case 3: after soft failure, negative CVS, LRS, on state
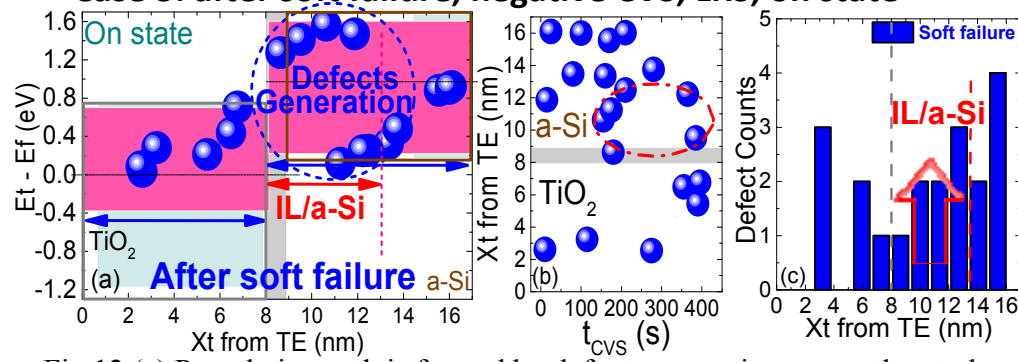

Case 4: region2 steep slope Negative CVS

Fig.12 (a) Percolation path is formed by defects generation across the stack after soft BD at Negative CVS. (b\&c) Defects generation found near IL/a-Si.
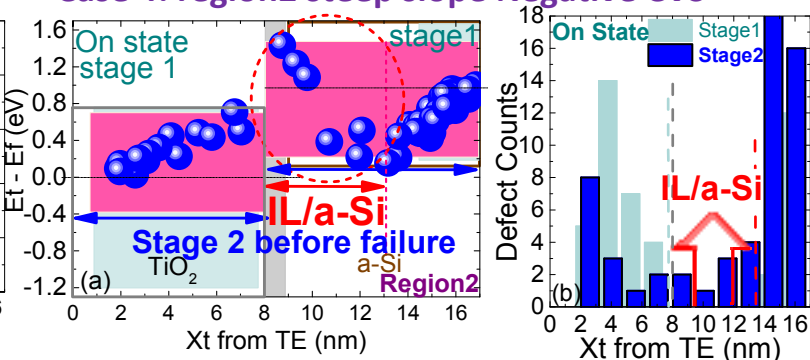

Fig.13 (a) Defects distribution before failure at short tBD in devices in Region 2 (steep slope) of negative CVS (Fig.4b). (b)

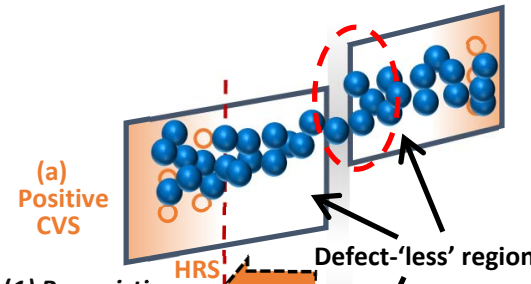

(1) Pre-existing defects profiles Defect-'less' regions

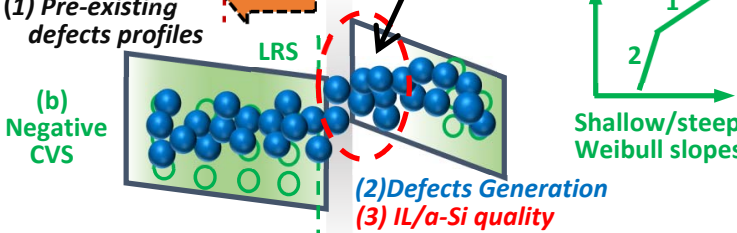

Fig14. Illustration of the switching and failure model.
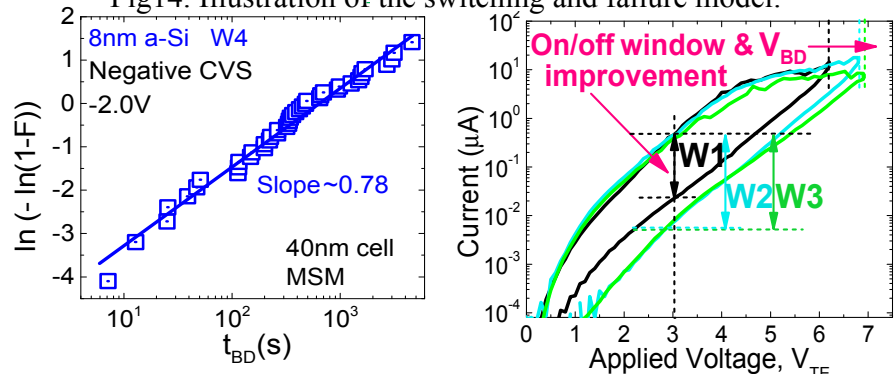

Fig15. tBD Weibull distribution of Fig.16 Memory window and single amorphous Si layer (MSM). $\mathrm{V}_{\mathrm{BD}}$ enhancement in W2\&3.
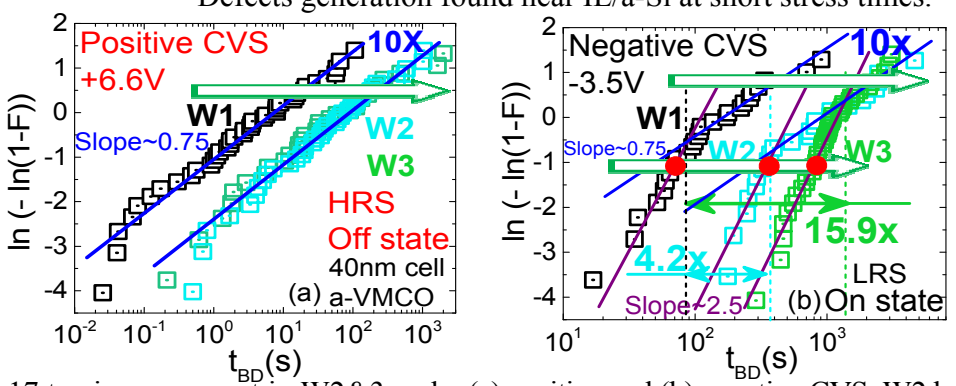

Fig. 17 tBD improvement in W2\&3 under (a) positive and (b) negative CVS. W2 has $10 \mathrm{x}$ larger $\mathrm{t}_{\mathrm{BD}}$ failure margins than $\mathrm{W} 1$ under both $\pm \mathrm{CVS}$. Further improvement in region 2 under -CVS is observed in $\mathrm{W} 3$ due to improved $\mathrm{TiO}_{2} / \mathrm{IL}$ quality.
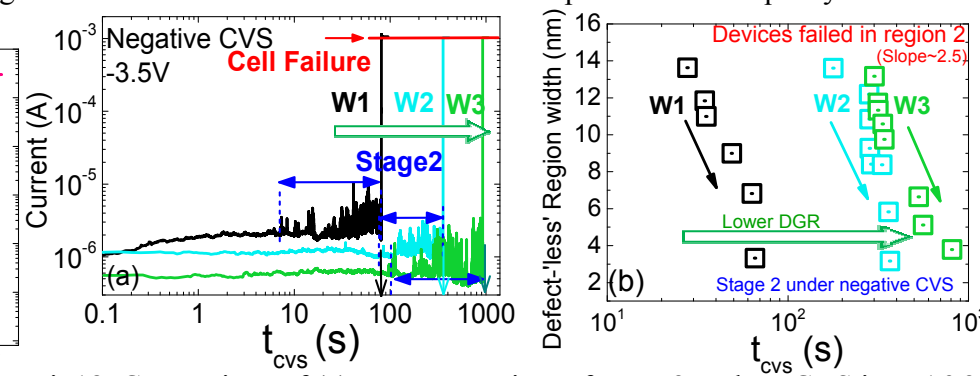

Fig18. Comparison of (a) occurrence time of stage 2 under - CVS in W1\&2\&3. (b) Progressive reduction of the generated defect-'less' region width against stress time in devices failed in region 2 as indicated by ' $\bullet$ 'symbols in Fig. 17 b. 